Bol. Acad. peru. leng. 63. 2018 (77-96)

\title{
EL LEGADO LINGÜÍSTICO DEL FUJIMORISMO ${ }^{1}$
}

\section{THE LINGUISTIC LEGACY OF FUJIMORISM}

\author{
Marco Antonio Lovón Cueva \\ Universidad Nacional Mayor de San Marcos
}

\section{Resumen:}

El fujimorismo ha sido estudiado desde los ámbitos de la política, la historia y la sociedad, pero no desde la lingüística. Esta investigación tiene por objetivo describir las palabras que se han creado en torno al fujimorismo. Desde los noventa, han aparecido voces vinculadas con las acciones, las decisiones y las posturas políticas de Alberto Fujimori y su familia, las cuales han ingresado al vocabulario popular, coloquial y estándar de los peruanos. Tales palabras forman parte de la memoria lingüística de los hablantes. Este trabajo es de corte lexicográfico, por tanto, recoge, enlista y explica dichas voces. Finalmente, en este estudio se concluye que el fujimorismo ha dejado un legado lingüístico variado en la memoria de los hablantes nacionales.

\section{Abstract:}

Fujimorism has been studied from the spheres of politics, history and society, but not from linguistics. This research aims at describing the words

\footnotetext{
1 Este artículo se ha elaborado en el marco del proyecto de investigación E18151751 del Vicerretorado de Investigación y Posgrado de la UNMSM.
} 
that have been created in connection with Fujimorism. Since the 90s, words linked to the actions, decisions and political positions of Alberto Fujimori and his family have appeared, which have been incorporated into the popular, colloquial and standard vocabulary of Peruvians. These words are part of the linguistic memory of the speakers. This paper is a lexicographic study; therefore, it collects, lists and explains these words. Finally, this study concludes that Fujimorism has left a varied linguistic legacy in the memory of national speakers.

Palabras clave: Fujimori; fujimorismo; léxico; lexicografía peruana; historia.

Key words: Fujimori; fujimorism; lexicon; Peruvian lexicography; history.

Fecha de recepción:

Fecha de aceptación:
$31 / 03 / 2018$

$31 / 05 / 2018$

\section{Introducción}

Así como existe una memoria histórica, existe una memoria lingüística. En el 2011, los peruanos crearon la palabra «PPKeiko» para calificar el apoyo político que Pedro Pablo Kuczynski (PPK) brindó abiertamente a Keiko Fujimori en el mitin de cierre de su campaña, después de que él no lograra pasar a segunda vuelta presidencial. Para entonces, Keiko Fujimori se enfrentaba a Ollanta Humala. En el mitin, PPK aseguraba que «queremos una economía estable. Y Keiko sí puede». En el 2016, el postulante de Peruanos por el Kambio sostuvo que se equivocó al brindar su apoyo a la líder de Fuerza Popular aquel año. Para el 2016, PPK logró llegar a segunda vuelta en competencia con Keiko Fujimori, a quien le ganará. Fue el año electoral en el que buscó desligarse de cualquier relación con ella; intentó borrar esa asociación lingüística: la de «PPKeiko». 
En general, los hablantes suelen recordar el pasado político a través de la poesía, la música, la novela y la lírica en general. Ejemplo de ello es la cita textual que empleó Keiko Fujimori del verso poético de Nicomedes Santa Cruz, que fue escrito en 1959, y que fue usado en el debate electoral del 2016 para recordarle a Pedro Pablo Kuczynski el respaldo que él le había brindado en el 2011, el cual reza así: «Cómo has cambiado pelona», verso que también fue utilizado por Kuczynski, pero en sentido contrario: «Tú no has cambiado pelona, eres la misma». Su intención era construir una imagen negativa y de rechazo hacia la candidata. Tanto Fujimori como Kuczynski apelaban a su memoria histórica y lingüística.

Es la lengua la que se convierte en el diccionario de las actividades y acontecimientos políticos, culturales, deportivos, sociales, que sus hablantes rememoran. Uno de los periodos políticos que ha generado la creación de palabras vinculadas con movimientos e inclinaciones políticas ha sido el de Alberto Fujimori, su gobierno, su periodo y sus acciones son recordados a través del léxico. Estas palabras no solo son usadas en las conversaciones cotidianas, sino también son empleadas en los medios de comunicación y en las redes sociales, en los cuales se crean nuevas palabras, es decir, neologismos, para referirse a los Fujimori. El impacto de Alberto Fujimori y el fujimorismo ha sido tanto que en el castellano peruano el apellido Fujimori se ha acortado a un elemento compositivo fuji-para crear nuevas palabras, tanto que se comporta como un prefijo en voces como fujicaviar o fujiaprismo. En otras casos, expresiones como Ley Fuji se han reducido a fujiley.

En este artículo, se recogen dichas voces y se presentan sus definiciones. Consideramos importante compilar este registro lingüístico en un trabajo descriptivo, dado que no existe hasta ahora un estudio lexicológico y lexicográfico que reúna y ordene en un solo listado todas estas palabras, sobre todo, de uno de los periodos de gobernanza más extensos de la República peruana y que tiene continuidad en la vida política del país. 


\section{El fujimorismo}

Los periodos de gobierno más extensos en el mundo suelen ser estudiados desde los ámbitos de la política, la economía, la historia y la sociedad, sobre todo en su relación con la democracia, la estabilidad económica, el cambio histórico y la rendición de cuentas. Sin embargo, pocos son estudiados desde el ámbito de la lingüística, más aún cuando determinados gobiernos generan crisis políticas y sociales que se reflejan en el léxico o habla cotidiana de los ciudadanos (Lovón y Pita, 2016). Uno de estos periodos escasamente estudiados por los lingüistas, específicamente, por los lexicógrafos, a pesar de que el fujimorismo ha encaminado la política y el habla peruana desde 1990, es el de la familia Fujimori. Los jóvenes de ahora entienden la derecha y la izquierda política sobre la base del fujimorismo.

El fujimorismo es posicionado en la derecha popular; está definido por su conservadurismo social y su visión neoliberal de lo económico, cuyas medidas fueron tomadas por Alberto Fujimori al inicio de su gobierno. Para el fujimorismo, el Estado cumple un rol mínimo en la economía; así, se sugiere privatizar las empresas estatales y aceptar la inversión extranjera. El impulso de tales medidas tiene su correlato en la política. Para el 5 de abril de 1992, el fujimorismo inicia su lado oscuro. Alberto Fujimori dio un autogolpe: cerró el parlamento por obstruir su mandato.

Según Tanaka (1998: 221), la consolidación del autogolpe estaba vinculada con signos de estabilidad y expectativas de mejoras. «Fue la estabilidad económica la que legitimó el autogolpe de abril de 1992, y no al revés. Así se legitimó y consolidó el "fujimorismo", y correlativamente, se descolocaron y colapsaron los partidos y el sistema partidario de los años ochenta». Para este autor, Fujimori tuvo éxito al estabilizar la economía y desarticular el modelo de desarrollo planteado en torno al Estado en tránsito hacia uno orientado hacia el mercado.

Para mantenerse en el gobierno, según Crabtree (2000: 65), Alberto Fujimori empleará prácticas populistas, con un estilo de gobierno personalista, alejado de las instituciones representativas y los sistemas formales de fiscalización (accountability); asimismo, relegará a los partidos 
políticos y forjará una relación nueva con el Ejército. Para este mismo autor (2000: 65), «[d]ebido a las nuevas políticas económicas y al impacto que tuvieron en la distribución y la desigualdad, se estableció un nuevo sistema de control político basado no en la inclusión y participación sino en nuevas formas de patrocinio y clientelismo. En este sentido, entonces, parece apropiado hablar de "neopopulismo" como un tipo de respuesta tradicional a nuevas condiciones». Los ciudadanos solo recibían, eran espectadores y consumidores; no había una participación propia o con voluntad.

En las elecciones, el fujimorismo aparecía para ganar en las urnas, pues los tradicionales partidos políticos estaban cuestionados. Su llegada al gobierno operaba junto con el apoyo de los militares, la policía y los empresarios. De acuerdo con López (2001: 169), el fujimorismo es una forma de representación política plebiscitaria que aparece en situaciones de crisis partidaria y que se apodera de la sociedad. Asimismo, el fujimorismo es un tipo de régimen político autoritario y tecnocrático que apela a los poderes fácticos como las fuerzas armadas y los organismos empresariales para hacer gobierno. El fujimorismo, asimismo, se adjudicará como logros para la reactivación económica, la derrota del terrorismo y la paz con el Ecuador.

Su éxito, no obstante, estará ligado con corrupción, sobre todo, de las instituciones públicas y privadas como la Policía, las escuelas, las universidades, los medios de comunicación, el medio de transporte, etc., que deteriorarán la calidad de vida, la seguridad y la confianza que depositaban los ciudadanos peruanos en tales instituciones. Según Marcus-Delgado y Tanaka (2001: 60-61), «el fujimorismo pretendió vana y desesperadamente mantenerse en el poder, pero terminó colapsando ante las múltiples evidencias de corrupción de un régimen autoritario que devino en una mafia inserta en el poder». En este panorama, Vladimiro Montesinos cumplió un papel crucial para mantener los gobiernos de Fujimori.

Asimismo, detrás del liderazgo de Alberto Fujimori, los miembros de su entorno familiar han movilizado el fujimorismo. Entre las principales figuras, destaca su hija, Keiko Sofía Fujimori Higuchi, quien se desempeñó como primera dama durante el régimen de su padre. Ella 
llegará a ser congresista de la República durante el periodo 2006-2011 con la más alta votación (más de 600000 votos según recuento oficial de la ONPE). Y será candidata presidencial en las elecciones generales del Perú de los años 2011 y 2016, en las cuales quedó en segundo lugar en ambos años. El otro miembro familiar que congrega a los simpatizantes del fujimorismo es su hijo Kenji Gerardo Fujimori Higuchi, quien ha sido congresista de la República desde 2011. Destaca de él que en las elecciones parlamentarias de Perú de 2016 fuera el candidato más votado con 326037 votos.

El rol de Keiko Fujimori ha sido crucial para el fujimorismo. Mientras su padre respondía ante la justicia, Keiko construyó un partido, alejado, principalmente, de los albertistas. Ella dirigirá como presidenta el partido Fuerza Popular, conocido inicialmente como Fuerza 2011, el cual logrará la mayoría parlamentaria para el periodo 2016-2021 con una bancada conformada por 73 representantes en un Congreso de 130 curules. Sin embargo, la personalidad de Keiko, así como el autoritarismo, el obstruccionismo y los errores generados por ella y por su propia bancada harán que pierda la mayoría absoluta. Su declive también está asociado con supuestos cargos de lavado de activos provenientes de la empresa Odebrecht para su campaña presidencial de 2011. Keiko junto con un alto número de políticos peruanos (Alejandro Toledo, Alan García, Ollanta Humala, Pedro Pablo Kuczynski) fueron envueltos en actos de corrupción relacionados con dicha constructora brasileña. Ella fue recluida en el Penal de Mujeres de Chorrillos tras un mandato de prisión preventiva de tres años.

Respecto de Kenji, es necesario resaltar el liderazgo que ha ido tomando en el fujimorismo. Él, junto con otros nueve congresistas disidentes, se marchó de Fuerza Popular en enero de 2018, y formó un bloque en apoyo de Pedro Pablo Kuczynski. Kenji evitó que proceda el pedido de vacancia presidencial. Esto le costó la enemistad con su hermana y la bancada, pero movilizó el pedido de indulto para su padre. En junio de 2018, Kenji junto con dos miembros de su bloque serán suspendidos del Congreso por 120 días con los votos del grupo consolidado en Fuerza 
Popular, al ser acusados de haber negociado favores políticos para evitar la destitución de Kuczynski. En medio de esta pugna, Kenji Fujimori ha mostrado un despliegue de una postura política menos conservadurista y más liberal, en contraste con Keiko.

Las actuaciones y los comportamientos políticos del fujimorismo cobran vida en el habla popular, coloquial y estándar de los peruanos, léxico que amerita ser registrado como una muestra entre política y lenguaje, entre la memoria política y la memoria lingüística.

\section{Metodología}

Siguiendo los lineamientos de Porto Dapena (2002), las palabras se enlistan en fichas lexicográficas: se presenta el lema, la información morfológica y la definición. Asimismo, se utilizan determinadas notas lexicográficas referidas al origen de la palabra, a una explicación histórica o al estudio que se haya hecho por otros lexicógrafos sobre el lema. Los lemas están ordenados por campos temáticos y en orden alfabético.

Los datos han sido comparados con el Diccionario de americanismos (ASALE, 2010), el Diccionario de la lengua española (RAE, 2014), el Diccionario de peruanismos (2009), de Álvarez Vita, y el Diccionario etimológico de palabras del Perú (2014), de Calvo, para corroborar el tratamiento que reciben las palabras.

\section{Análisis lexicográfico}

\subsection{Palabras vinculadas con Alberto Fujimori}

albertismo [sustantivo]. Tendencia política que promueve el gobierno y el liderazgo de Alberto Fujimori.

albertista [adjetivo]. Seguidor o partidario de Alberto Fujimori.

antishock [sustantivo]. Opción política que propone evitar la aplicación de un programa de ajuste de precios. 
Nota: En junio de 1990, Alberto Fujimori prometió no aplicar el shock económico propuesto por la coalición política Frente Democrático (Fredemo). Sin embargo, lo aplicó. Dicha promesa se conoce como el «antishock».

autogolpe [sustantivo]. Disolución del ente legislativo, parlamento o Congreso de la República, realizada por parte del presidente o jefe de Estado para hacerse con el poder y consolidarse en el cargo.

Nota: De acuerdo con la RAE (2014), la palabra «autogolpe» está definida como 'violación de la legalidad vigente en un país por parte de quien está en el poder, para afianzarse en él'. Antes de los noventa, la palabra no figuraba en el diccionario de la RAE. Posteriormente, ingresó al diccionario.

efecto Tiwinza [sustantivo]. Resultado favorable, particularmente, político y electoral, que se obtiene a partir del cierre fronterizo con Ecuador.

Nota: El conflicto del Alto Cenepa que enfrentó al Perú con Ecuador entre los meses de enero y marzo de 1995 culminó con la adjudicación de victoria por parte de ambos países. Los países garantes resolvieron que la demarcación de la frontera sería la misma que fue establecida en 1942. Es decir, se ratificó la posesión de la zona del Cenepa, en la que se incluye Tiwinza. Sin embargo, a la vez, se estipuló otorgar un kilómetro cuadro de Tiwinza en condición de propiedad privada a Ecuador, sin perjuicio de la soberanía del Perú, para realizar actos conmemorativos, pues en dicha área se encuentran sepultados 14 soldados ecuatorianos. La aparente victoria, o empate, para el Perú fue empleada por Alberto Fujimori para atraer a la ciudadanía y respaldar su candidatura para las elecciones de 1995.

electroshock [sustantivo]. Tratamiento psiquiátrico que induce estados de coma a través de descargas eléctricas.

Nota: La RAE (2014) registra la palabra «electrochoque» en su diccionario y la define como 'tratamiento de una perturbación mental provocando el coma mediante la aplicación de una descarga eléctrica'. En el Perú, se popularizó el vocablo «electroshock» debido a las declaraciones de Susana Higuchi sobre este método psiquiátrico al que era expuesta. El 29 de octubre de 2001, Higuchi afirmó: «Sufrí tortura con electroshock en dos oportunidades: una en 
el año 1992 y otra en el año 2000. El electroshock fue luego de la denuncia de la ropa donada, luego del autogolpe, y dentro de esos cuatro meses que me mantuvieron encerrada en el Pentagonito, en el Servicio de Inteligencia del Ejército, me torturaron con electroshock y de las cuales todavía tengo, pueden ser visibles ciertas huellas de quemadura hasta en la cara y en todo el cuerpo. Quizás mayo de 1992. Y la otra fue específicamente el domingo 4 de junio del año 2000, en la UCI (Unidad de Cuidados Intensivos) del Hospital Loayza, cuando ya era congresista electa, mas no congresista juramentada». El plural de esta palabra es «electroshocks».

esterilizaciones forzosas [colocación]. Conversión de alguien en estéril sin consentimiento y sin justificación médica o clínica, generalmente, con el fin de impedir la reproducción de un grupo social o étnico.

Nota: También se emplean las colocaciones «esterilización forzada», «esterilización masiva», «esterilización compulsiva». Ninguna de estas colocaciones está registrada en el diccionario de la RAE (2014). Entre 1996 y 2001, según la Defensoría del Pueblo, se realizaron 272 mil 028 operaciones de ligaduras de trompas y vasectomías: «La Defensoría del Pueblo constató que se realizaron 272 mil 028 operaciones de ligaduras de trompas y vasectomías entre 1996 y 2001, pero se desconoce cuántas se ejercieron mediante presuntos engaños y coacciones» (La Prensa, 2015).

fujimontesinismo [sustantivo]. Conexión política entre el fujimorismo y el montesinismo que entraña prácticas dictatoriales, de corrupción y de violación de derechos humanos.

Nota: Según Calvo (2014), el «fujimontesinismo» es el 'periodo de la historia del Perú que comprende los gobiernos de Fujimori y Montesinos'.

fujimontesinista. 1. [sustantivo]. Persona inclinada a las maniobras planificadas y ejecutadas por el fujimontesinismo. 2. [adjetivo]. Relacionado con el fujimontesinismo.

Nota: Para Álvarez Vita (1999), «fujimontesinista» 'se dice del régimen de gobierno de Alberto Fujimori, presidente del Perú, y de su asesor Vladimiro Montesinos'. Calvo (2014) define «fujimontesinista» como 'relativo al fujimontesismo'. 
fujimorada [sustantivo]. Acto antidemocrático o pseudodemocrático.

Nota: Según Álvarez Vita (1999), «fujimorada» indica un 'hecho o actitud pseudodemócrata'.

fujimorato [sustantivo]. Periodo de gobierno presidencial de Alberto Fujimori, caracterizado por el empleo de prácticas autoritarias y dictatoriales.

Nota: Álvarez Vita (1999), define «fujimorato» como 'periodo comprendido entre el 28 de julio de 1990 y el 17 de noviembre de 2000, en que el ingeniero Alberto Fujimori ocupó la presidencia de la República’. Para Calvo (2014), el «fujimorato» es el «régimen presidencial de Fujimori». En el Perú, la palabra «fujimorato» se creó por analogía con «shogunato», que refiere al gobierno militar que tuvo Japón a fines del siglo xvir hasta la época de la Restauración Meiji de 1868.

fujimoriano [adjetivo poco usado]. Relacionado con Alberto Fujimori.

Nota: Álvarez Vita (2009) define «fujimoriano» como 'perteneciente o relativo a Alberto Fujimori, quien fue presidente de la República del 28 de julio de 1990 y el 17 de noviembre de 2000'.

fujimorismo [sustantivo]. Movimiento político de tendencia conservadora que se caracteriza por su pragmatismo político, cuya cabeza visible ha sido Alberto Fujimori.

Nota: Calvo (2014) define «fujimorismo» como el 'periodo de tiempo que comprende los gobiernos de Fujimori'. Para el politólogo Francisco Miró Quesada Rada (2014), «el fujimorismo no es una ideología explícita, porque no la tiene. Es, más bien, una forma de hacer política en que se mezclan caudillismo, populismo, clientelismo y abuso del poder».

fujimorista [sustantivo]. 1. Persona partidaria del fujimorismo. 2. [adjetivo]. Relacionado con el fujimorismo. Ej.: partido fujimorista.

Nota: Álvarez Vita (2009) define la palabra «fujimorista» como 'Persona partidaria de Alberto Fujimori, quien fue presidente de la República del 28 de julio de 1990 y el 17 de noviembre de 2000'. Según Calvo (2014), «fujimorista» refiere a «Partidario de Fujimori». 
fujiprensa [sustantivo]. Agrupación de medios de comunicación controlados por el gobierno de Alberto Fujimori, dirigidos para difundir principalmente sus obras y acciones.

Nota: Para Álvarez Vita (2009), la «fujiprensa» se refiere a los 'medios de difusión adictos al ingeniero Alberto Fujimori, quien fue presidente de la República del 28 de julio de 1990 y el 17 de noviembre de 2000’.

fujishock [sustantivo]. Programa de ajuste de precios aplicado por Alberto Fujimori para reducir la inflación y reactivar la economía.

Nota: El «shock económico» se aplicó para corregir los desequilibrios económicos producidos por los subsidios insostenibles y la hiperinflación galopante dejados por el primer gobierno de Alan García.

geisha [sustantivo]. Mujer, particularmente periodista, cercana a Alberto Fujimori y a su entorno político íntimo, quien respalda su gobierno e, incluso, lo acompaña en sus viajes y entrevistas.

Nota: Para Sifuentes (2009): «En la década de los 90 la prensa se vendió mediante una mecánica sencilla: copiar y pegar. Había una central en el SIN que proveía de información a la mayoría de medios y esa era la única fuente admisible para los periodistas que se pusieron el kimono. Las geishas copiaban y pegaban lo que les daba Montesino y listo. ¿Quién se iba a tomar la molestia de cruzar la información, de corroborar si lo que dijeron era verdad o no? Nadie. No había cómo».

higuchista [adjetivo desusado]. Seguidor de Susana Higuchi.

Nota: Susana Igushi se presentó, con su agrupación «Armonía Frempol», como candidata al Congreso de la República en 1995, pero su inscripción fue invalidada por el poder electoral. El 2 de febrero de 1995, Higuchi denuncia a la República del Perú ante la Comisión Interamericana de Derechos Humanos porque el «Jurado Nacional de Elecciones había violado, en perjuicio de ella, el artículo 23 de la Convención Americana sobre Derechos Humanos al impedirle, en forma arbitraria e ilegal, que se postulase» (CIDH, 1999). Posteriormente, la Comisión Interamericana de Derechos Humanos confirma que se había violado derechos de participación en dicha elección. Susana Higuchi, en los años 2000 y 2001, postulará y será electa como congresista. $\mathrm{El}$ adjetivo fue brevemente usado en los noventa. 
jueces sin rostro [sustantivo]. Fiscales y jueces que, según la Ley Antiterrorista de 1992, juzgan a acusados de violencia política, principalmente de terrorismo, quienes cubren su rostro para no ser amenazados de muerte.

meter la yuca [locución verbal]. Engañar, mentir, estafar a alguien.

Nota: También se usa «enyucar». La expresión «meter la yuca» se encuentra en el Diccionario de americanismos (ASALE, 2010). Esta locución verbal se popularizó cuando los medios de comunicación recordaron una escena televisiva en la que Alberto Fujimori aparecía levantando el tubérculo como una acción simbólica de vincularse con la población y cumplir con sus promesas.

montesinismo [sustantivo]. Prácticas de espionaje, infiltración, secuestro, tortura, asesinato selectivo o corrupción aplicadas por Vladimiro Montesinos en su cargo de asesor presidencial y jefe del Servicio de Inteligencia Nacional del Perú.

Nota: A Vladimiro Montesinos se le llegó a conocer como la «eminencia gris»; el «doc», por «doctor»; y «Rasputín» (El Comercio, 2001) o «Rasputín de los Andes». Algunas colocaciones en las que aparece la palabra «montesinismo» son las siguientes: tentáculos del montesinismo, nexos con el montesinismo, vínculos con el montesinismo.

montesinista [sustantivo]. 1. Persona que aplica el montesinismo. 2. [adjetivo]. Relacionado con el montesinismo.

Nota: Algunas colocaciones en las que aparece la palabra «montesinista» son las siguientes: mafia montesinista, psicosocial montesinista, red montesinista, operador montesinista.

prensa chicha [colocación]. Agrupación de medios de comunicación dedicados a la elaboración y reproducción de noticias sensacionalistas.

Nota: Otras colocaciones son «diario chicha», «periódico chicha» y «prensa amarillista». En el año 2011, Vladimiro Montesinos testimonió lo siguiente sobre los «diarios chicha»: «El Ingeniero Fujimori era consciente de que la prensa escrita, particularmente aquellos diarios que por su bajo precio 
llegaban a la opinión pública en forma masiva, producía el efecto de orientar la corriente de opinión. Es por ello que el expresidente Fujimori me ordena que se efectúe un estudio de medición pública sobre los grados y niveles de aceptación que tenían los «diarios chicha» [...] con la finalidad de tener un cabal y oportuno conocimiento sobre los medios con los que se tenía que trabajar [...]. Decidió que debería iniciarse un contacto con los diarios 'Mañanero', 'La Chuchi', 'Diario Más', 'El Chato', 'Conclusión', 'El Tío' y ‘La Yuca' [...]. Se paga a los periódicos con la finalidad de difundir la imagen de Fujimori y apoyar su campaña de reelección presidencial, así como cuestionar a los opositores políticos. Todo se hizo en beneficio exclusivo del expresidente Fujimori y por orden expresa del mismo es que se implementó esa tarea».

re-reelección [sustantivo]. Tercera elección consecutiva o inmediata, mientras se está aún en ejercicio del cargo, cuya habilitación legal depende de las normas e instituciones constitucionales.

Nota: Alberto Fujimori se presentó a las elecciones presidenciales de 1990, 1995 y 2000. Para su tercera postulación recurrió a la figura jurídica de la interpretación auténtica de la Constitución. Según la Constitución de 1993, el presidente solo puede ser reelegido para un periodo inmediato.

Sin embargo, de acuerdo con la interpretación presentada por Fujimori, él habría sido candidato en 1995 y, por tanto, su segunda postulación sería la del 2000, pues la constitución de 1993 no existía cuando se presentó en 1990; esta última no contaría.

salita del SIN [sustantivo]. Habitación del Servicio de Inteligencia Nacional en la que se trataron asuntos fujimontesinistas.

Nota: También conocida como el «salón gris» o sin diminutivo «sala del SIN». En esta sala, Vladimiro Montesinos se reunió, principalmente, con políticos, militares, empresarios y responsables de medios de comunicación.

tsunami Fujimori [colocación sustantiva]. Candidatura de Alberto Fujimori que arrasó con la de Mario Vargas Llosa en las elecciones de 1990.

vladivideo [sustantivo]. Video que compromete, principalmente, en actos de corrupción al asesor presidencial Vladimiro Montesinos. 
Nota: Álvarez Vita (2009) define «vladivideo» como 'video filmado con fines de registrar un hecho generalmente doloso o comprometedor'. Para Calvo (2014), «vladivideo» refiere a 'video que registra las actividades ilegales entre Montesinos y sus cómplices'. Al respecto, se precisa que el primer vladivideo se presentó en una conferencia de prensa. En este se mostró la entrega de fajos de dinero por parte de Vladimiro Montesinos al entonces congresista Alex Kouri, con el fin de que apoye y forme parte de la agrupación fujimorista. Posteriormente, en la casa de Montesinos se encontraron 700 videos; muchos de los cuales fueron confiscados por Alberto Fujimori. Los videos eran grabados en cinta de VHS en la sala del SIN.

\subsection{Palabras vinculadas con los descendientes de Fujimori}

avenger [sustantivo]. Bloque de congresistas fujimoristas que sigue el liderazgo de Kenji Fujimori.

Nota: Kenji Fujimori nombró avengers a los nueve congresistas fujimoristas que se abstuvieron en vacancia presidencial de PPK, desobedeciendo el acuerdo de la bancada. La voz pasó luego como nombre común en el vocabulario peruano para referirse a este grupo. The Avengers (Los vengadores) es una película americana de superhérores en la que se recluta a diversos héroes para formar un equipo que defienda y evite la destrucción de la tierra. El grupo tenía como premisa «hacer frente a los enemigos que ningún héroe podría derrotar solo». Kenji en su Twitter@ kenjiFujimoriH, escribió «Hoy me reuní con los "Avengers", los héroes que salvaron la democracia y cambiaron la historia. Mi eterno agradecimiento por su valentía». Su apoyo a PPK favoreció que kuczynski después proceda a otorgarle el indulto a su padre Alberto Fujimori.

fujicoctel [sustantivo]. Reunión social organizada por el partido fujimorista en la que los invitados aportantes contribuyen económicamente con el pago de la invitación para recaudar fondos de financiamiento para la campaña electoral.

Nota: Fuerza Popular ha organizado distintos cocteles. Uno de ellos es el coctel privado del 21 de diciembre de 2015 en el que se recaudó 710419 soles. El plural de esta voz es «fujicocteles». 
fujirrifa [sustantivo]. Sorteo de premios organizado por el partido fujimorista en el que los compradores aportan con el pago del boleto con el fin de que se recauden fondos de financiamiento para la campaña electoral.

Nota: La rifa organizada por Fuerza 2011, en el año 2011, recaudó 1500 000 soles como fondo de campaña. De los 13 ganadores solo se presentó uno a recoger su premio: un horno microondas. Asimismo, entre otras premiaciones, estaba un auto $0 \mathrm{~km}$, que no fue entregado, porque el ganador nunca acudió a reclamarlo. Este suceso motivó a la prensa, y junto con los usuarios de las redes sociales, a crear y reproducir la voz «fujirrifa». El plural de esta voz es «fujirrifas».

fujitáper [sustantivo]. Recipiente de plástico cuya tapa lleva el logotipo del partido Fuerza Popular y se otorga a la población con el fin de que inclinen su voto por esta agrupación política.

Nota: El táper sirve para promover propaganda electoral a favor de Fuerza Popular. El contenedor plástico puede contener cajas de fósforos, bolsas de sopa instantánea, bolsa de gelatina en polvo, comida preparada (arroz con pollo) o dinero (billetes de diez nuevos soles) (El Diario de Curwen, 2016). También se usa la expresión el «táper naranja».

fujitroll [sustantivo]. Simpatizante del fujimorismo que arremete contra cualquier opositor de este movimiento a través de las redes sociales.

Nota: Los usuarios identificados como «fujitrolls» usaron el Twitter para manifestar su apoyo político; sin embargo, hubo mensajes cargados de agravios cuando se referían a la prensa o agrupaciones políticas antagonistas: «Una polémica se ha desatado en las redes por la identificación de varios usuarios de Twitter. Estos han sido llamados «fujitrolls», debido a sus comentarios beligerantes y su afinidad con el fujimorismo» (La República, 2016). Uno de ellos pidió disculpas por sus actos. Frente a todo esto, el nuevo vocablo se extendió a través de las redes sociales. El plural de esta voz es «fujitrolls».

fujiwar [sustantivo]. Disputa entre los hermanos Fujimori por la candidatura a la presidencia de la República.

Nota: Esta palabra está formada por el acortamiento de la voz «Fuji(mori)» $\mathrm{y}$ «war» 'guerra' en inglés. 
keikismo [sustantivo]. Variante política del fujimorismo, cuya lideresa es Keiko Fujimori.

Nota: Keiko Fujimori dirige el partido Fuerza Popular, antes llamado Fuerza 2011 (el nombre fue cambiado en el 2012).

keikista [sustantivo]. 1. Persona partidaria de Keiko Fujimori. 2. [adjetivo]. Relacionado con el keikismo.

Nota: Algunas colocaciones en las que aparece la palabra «keikista» son las siguientes: partido keikista, vertiente keikista, repunte keikista.

keikovideo. [sustantivo]. Video que graba prácticas de corrupción, y cuya grabación se imputa a Keiko Fujimori.

Nota: Kenji Fujimori fue grabado por uno de los miembros de su bancada, Moisés Mamani, en el momento en el que le ofrecía privilegios con el Ejecutivo. Dicho video facilitó que Keiko Fujimori y la bancada consolidada denuncien públicamente las prácticas de corrupción atribuidas a Kenji Fujimori y su bloque. En analogía con los vladivideos, en el que se filmaba a los funcionarios que Montesinos corrompía, se creó el término keikovideo, como si ella fuera quien dirigiese las grabaciones.

kenjista [adjetivo]. Seguidor de Kenji Fujimori.

kenjivideo. [sustantivo]. Video que compromete, principalmente, a Kenji Fujimori en actos de corrupción, como cohecho pasivo activo y tráfico de influencias.

Nota: Kenji Fujimori junto con sus compañeros parlamentarios Bienvenido Ramírez y Guillermo Bocángel fueron grabados por Moisés Mamani, otro miembro de Fuerza Popular, en un momento en el que le ofrecían que sus proyectos iban a tener acceso privilegiado con el Ejecutivo a cambio de votar en contra en el segundo pedido de vacancia presidencial contra Pedro Pablo Kuczynski. El grupo de videos filmados que desfavorecían a Kenji se conocen como kenjivideos.

naker [sustantivo]. Persona que se opone a la candidatura de Keiko Fujimori. 
Nota: Esta palabra es derivada de las siglas NAK (No a Keiko), a las que se ha añadido un sufijo nominalizador «er». El plural es «nakers».

naranja 1. [sustantivo]. Partidario del fujimorismo. 2. [adjetivo]. Relacionado con el fujimorismo.

Nota: Algunas colocaciones en las que aparece la palabra «naranja» son las siguientes: tendencia naranja, agrupación naranja. También se emplea la palabra con el diminutivo: «naranjita». Ej.: Él es naranjita, naranjita.

\subsection{Palabras creadas en torno al fujimorismo}

fujiaprista 1. [sustantivo]. Persona inclinada a las maniobras planificadas y ejecutadas por el fujiaprismo. 2. [adjetivo]. Relacionado con el fujiaprismo.

fujiaprismo [sustantivo]. Alianza política entre Fuerza Popular y el APRA.

fujicaviar [adjetivo despectivo]. Antiguo defensor del fujimorismo que en la actualidad preconiza ideas de la izquierda, generalmente, para beneficiarse de ellas.

fujimorizar [verbo transitivo]. Convertir a alguien al fujimorismo.

fujimorizarse [verbo pronominal]. Inclinarse por el fujimorismo.

fujirrata [adjetivo despectivo]. Dicho de un fujimorista: infame y despreciable.

Nota: Este vocablo es empleado con connotación negativa y está formado por el lexema fuji- y rata. En el Perú, la rata es un animal asociado con corrupción y abominación. Así, a Castañeda Lossio lo apodaron «Ratañuela» y a Alan García «Ratalán», como una manera de evidenciar su rechazo a estos políticos $\mathrm{y}$ a sus procederes.

neofujimorismo [sustantivo]. Fujimorismo renovado o reformado. 
neofujimorista [adjetivo]. Relacionado con el neofujimorismo.

\section{Conclusiones}

En este artículo, se han recogido las palabras que se han creado en torno a Alberto Fujimori y a su familia (sustantivos, adjetivos, verbos). Este estudio lexicográfico reúne las voces de uno de los periodos políticos que ha calado en la memoria colectiva de la sociedad.

Al respecto, sería interesante recoger otras voces productivas vinculadas con los diversos periodos políticos peruanos y saber más de ellas, sus significados, sus marcas gramaticales, su extensión de uso. Hacer un trabajo lexicográfico es una tarea compleja de realizar; por ejemplo, sobre este trabajo, es necesario preguntarse: ¿Qué otras expresiones lingüísticas se pueden recoger? ¿Siglas, frases, letras de canciones? Los periodos políticos de gobernanza extensos por lo general producen y reproducen variedad de vocablos; por ello, están vigentes en el recuerdo histórico-lingüístico. Y el fujimorismo ha dejado un legado lingüístico en los hablantes nacionales. 


\section{BIBLIOGRAFÍA}

ÁlVAREZ VITA, J. (2009). Diccionario de peruanismos. El habla castellana del Perú. Lima: Academia Peruana de la Lengua y Universidad Alas Peruanas.

ASALE (Asociación de Academias de la Lengua Española) (2010). Diccionario de americanismos. Madrid.

CALVO, J. (2014). Diccionario etimológico de palabras del Perú. Lima: Universidad Ricardo Palma.

CRABTREE, J. (2000). «Neopopulismo y el fenómeno Fujimori». Crabtree, John y Jim Thomas (editores). El Perú de Fujimori: 1990-1998. Lima: Universidad del Pacífico e IEP.

Comisión Interamericana de Derechos Humanos (1999). Informe N. ${ }^{\circ}$ 119/99. Caso 11, 428 Susana Higuchi Miyagawa, Perú. <http://bit.ly/1sXtba9>

El Comercio (25 de junio de 2001). El hombre más poderoso y corrupto de la bistoria del Perú. Lima. < http://bit.ly/1UeesND>.

El Diario de Curwen (8 de junio de 2016). «3 tipos de fujitáper que pude detectar a lo largo de la campaña». Utero.pe. <https://bit. ly/2V2J2C1>

La Prensa. Amnistía pide a Perú un registro de víctimas de las esterilizaciones forzadas. Lima: 21 de octubre de 2015. < http://bit.ly/1NFw6gC>

La República (13 de mayo de 2016). Twitter: Identifican a "fujitroll" y pide disculpas a periodistas. Lima. < http://bit.ly/1OYGRWn>

LÓPEZ, S. (2001). «El fujimorismo como régimen político; límites y perspectivas». Plaza, Orlando (editor), Perú. Actores y 
https://doi.org/10.46744/bapl.201801.003

Escenarios al Inicio del Nuevo Milenio. Lima: Fondo Editorial

- Pontificia Universidad Católica del Perú , pp. 169-206.

LOVÓN CUEVA, M. A. y GARCÍA PITA, P. S. (2016). «Los términos de la crisis venezolana». Boletín de Lingüística, vol. XXVIII, n. ${ }^{\circ} 45-$ 46, 79-110. <https://bit.ly/2TTzIjA >

MARCUS-DELGADO, J. y TANAKA, M. (2001). Lecciones del final del fujimorismo: la legitimidad presidencial y la acción política. Lima: IEP.

MIRÓ QUESADA RADA, F. (2014). «Qué es el fujimorismo?, por Francisco Miró Quesada Rada». El Comercio. Lima: 9 de octubre de 2014. <http://bit.ly/1t50Pcd>

PLANAS, P. (1999). El fujimorato. Estudio político-constitucional. Lima. $<$ http://bit.ly/1THKi62>

RAE (Real Academia Española) (2014). Diccionario de la lengua española. Madrid.

SANTA CRUZ, N. (1959). Cómo has cambiado pelona. Chincha. <http:// bit.ly/1qZ7X9N>

SIFUENTES, M. (12 de abril de 2009). «Geishas del siglo XXI». Perú21. Blog. Lima. <http://bit.ly/1THKiCU>

TANAKA, M. (1998). Los espejismos de la democracia: el colapso del sistema de partidos en el Perú. Lima: IEP. 